\title{
I 0 I 6 Increased sensitivity for detection of intra-cardiac thrombus using phase sensitive inversion recovery (PSIR) late enhancement techniques for combined myocardial scar and thrombus imaging Weale J Peter*1, George Lin ${ }^{2}$, Sven Zuehlsdorff1, Francois Christopher ${ }^{2}$, David Tuit ${ }^{2}$, Renate Jerecic ${ }^{1}$ and James Carr $^{2}$
}

Address: ${ }^{1}$ Siemens Medial Solutions, Chicago, IL, USA and ${ }^{2}$ Northwestern Memorial Hospital, Chicago, IL, USA

* Corresponding author

from I th Annual SCMR Scientific Sessions

Los Angeles, CA, USA. I-3 February 2008

Published: 22 October 2008

Journal of Cardiovascular Magnetic Resonance 2008, I0(Suppl I):AI4I doi:I0.II86/I532-429X-I0-SI-AI4I

This abstract is available from: http://jcmr-online.com/content/I0/SI/AI4I

(C) 2008 Peter et al; licensee BioMed Central Ltd.

\section{Introduction}

Late enhancement techniques have been recently used for the detection of intra-cardiac thrombus due to its characteristic appearance in DE images. For time reasons in clinical practice frequently a TI is chosen which nulls the normal myocardium and not the thrombus allowing simultaneous evaluation of myocardial scar and thrombus. This work demonstrates the pitfalls of solely magnitude based DE imaging for the simultaneous detection of intra-cardiac thrombus and shows the benefits of the PSIR technique by theoretical and clinical analysis

\section{Methods}

In Phase-Sensitive-Inversion-Recovery (PSIR) techniques the reconstructed image discriminates between positive and negative signal amplitudes. Thus PSIR reduces the need to set precise inversion times to avoid reduced or even inverted contrast between areas of hyper-enhancement and normal myocardium [1].

The same theoretical advantage can be used for the detection of intra-cardiac thrombus where the T1 is typically longer than any other cardiac structure in a contrastenhanced scan $[2,3]$. It is possible that in magnitude (non-PSIR) images acquired with a TI to null normal myocardium the signal intensity from thrombus is close to that of the contrast-enhanced blood-pool of the cardiac chambers leading to contrast loss between thrombus and surrounding blood. A simulation of the signal-time-curve at different TIs was performed using typical T1-values of $600 \mathrm{~ms}$ (Thrombus) and $300 \mathrm{~ms}$ (Contrast enhanced blood) to demonstrate this effect. To verify the theoretical advantages of PSIR an evaluation of the relative contrast (CI) between thrombus $\left(\mathrm{S}_{\mathrm{T}}\right)$ and blood pool $\left(\mathrm{S}_{\mathrm{BP}}\right)$ for both magnitude and PSIR images was performed $\left(\mathrm{CI}_{\mathrm{T}}=\right.$ $\left.\left(S_{T}-S_{B P}\right) / S_{B P}\right)$ in 36 patients with known intra-cardiac thrombus. These patients had undergone DE using a TIscout followed by a PSIR technique which concurrently reconstructs the corresponding magnitude and phase sensitive images. The TI was chosen to null the myocardium. The data of the TI-Scout were used to plot the signal evolution at different TI times (Figure 2) to verify the theoretical advantages seen in the simulation (Figure 1).

\section{Results}

The recovery curves in Figure 1 clearly demonstrate the advantage of the PSIR method. At a chosen TI (in this case $300 \mathrm{~ms}$ ) the difference between the signal amplitudes for thrombus and blood is substantially less than in the PSIR reconstruction. It is possible that for some combinations of TI, contrast-agent concentration and scan-timing that the thrombus/blood-pool contrast could be eliminated. The signal behaviour of the TI scout data in a patient with thrombus confirms the result of the simulation. The mean contrast index value was $\mathrm{CI}_{\mathrm{PSIR}}=1.07$ (S.D 0.86) for the PSIR method vs $\mathrm{CI}_{\mathrm{Mag}}=0.66$ (S.D 0.57) for the magnitude reconstruction. The qualitative contrast improvement is 


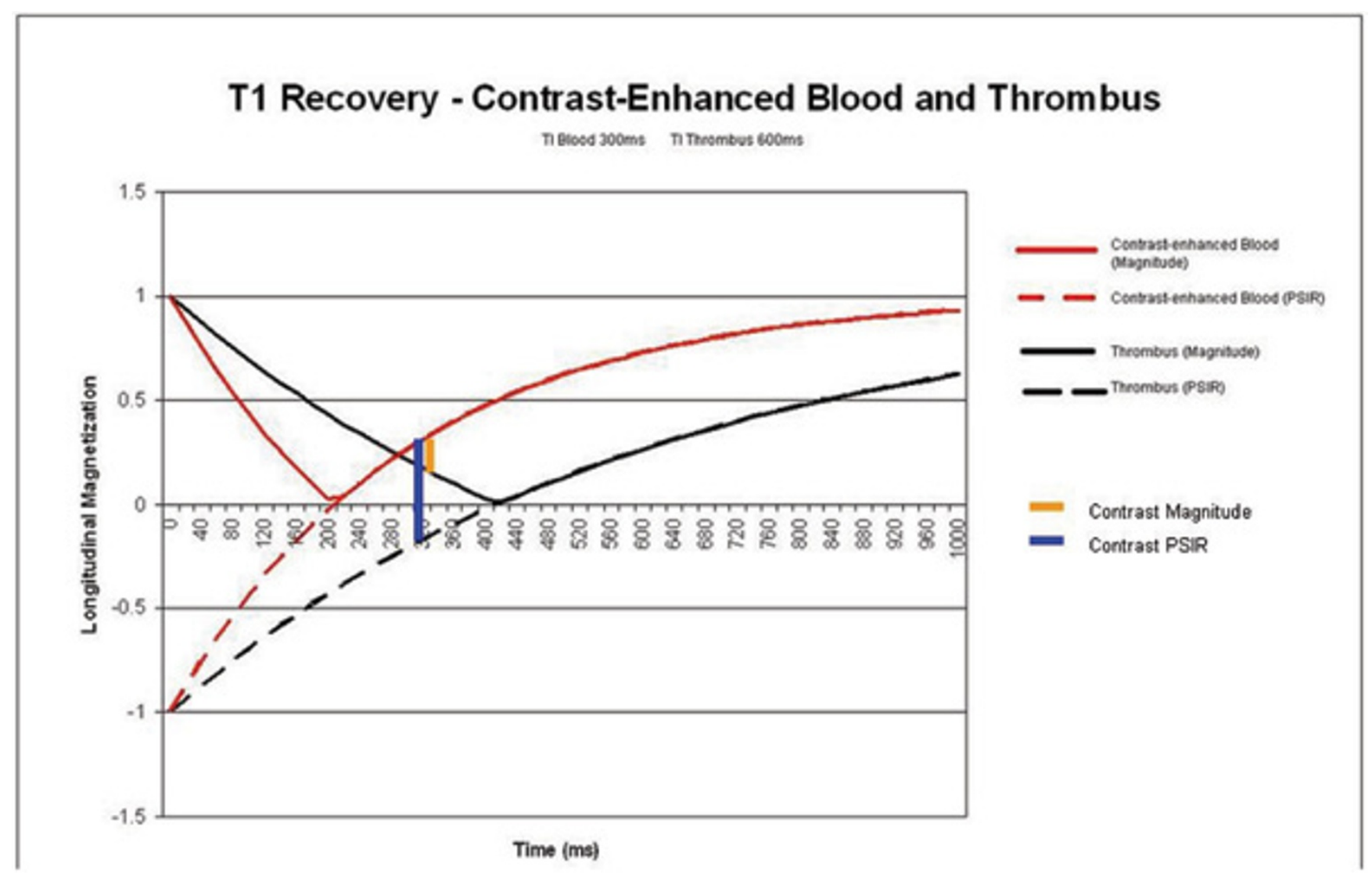

Figure I

Simulated TI Recovery curves for two different tissues with TI values similar to contrast-enhanced blood and thrombus. The region between the zero crossing points of the two tissues illustrates rapidly reducing and inverting contrast in the range of $\mathrm{TI}$ values commonly used in MDE imaging - this is eliminated with the use of a Phase Sensitive IR sequence which preserves true TI contrast.

shown in a direct comparison of both reconstruction methods (Figure 3).

\section{Conclusion}

The theoretical benefits in the use of a PSIR method as a robust method to depict intra-cardiac thrombus within a single DE study are validated in-vivo. In magnitude images the contrast between thrombus and contrastenhanced blood is compromised in the range of TI values typically used in DE to null the myocardium and small thrombi could remain undetected. Non-PSIR methods require additional scans with optimized TI times to maximize sensitivity to thrombus.

A pilot study recently described the use of DE techniques for detection of left atrial appendage thrombus [4] and reported a low sensitivity (44\%) using a non-PSIR technique. The range of TI values suggests that the optimization was for myocardial nulling which results in the compromised contrast illustrated above. The use of a
PSIR-technique should result in increased sensitivity in detection of LAA and other intra-cardiac thrombi without the need for an additional scan or optimization of TItimes.

\section{References}

I. Kellman P, et al:: MRM 2002, 47:372-382.

2. Rapoport, et al.: Radiology 1987, 162:527-530.

3. Schlosser, et al.: Radiology 2005, 236: I04 I-1046.

4. Mohrs O, et al.: AJR 2006, I 86: 198-205. 


\section{Signal Evolution in Contrast-Enhanced Blood and Thrombus (TI Scout sequence)}

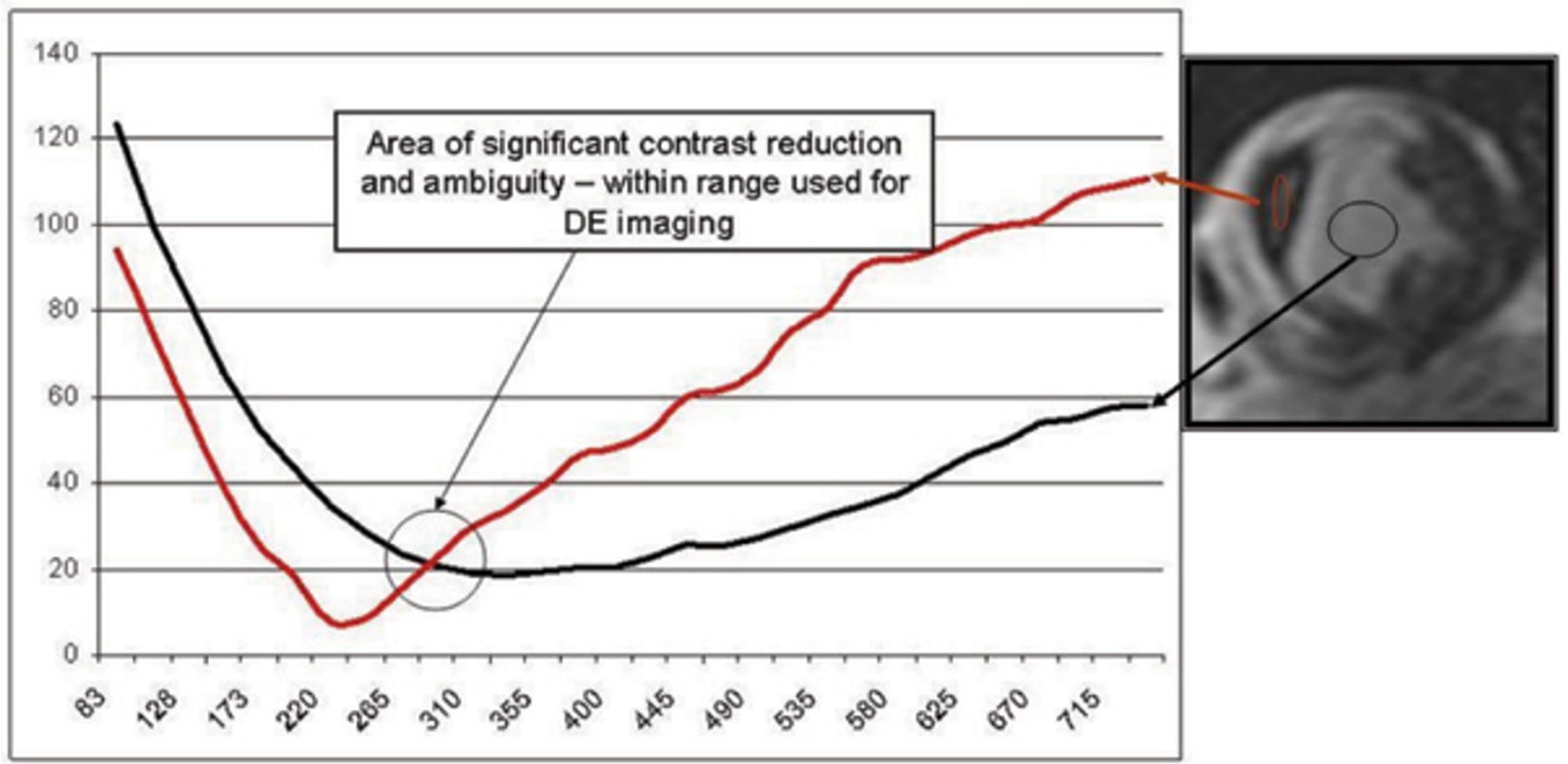

\section{Contrast Enhanced Blood}

\section{Thrombus}

Figure 2 Signal evolution with inversion time for blood pool and thrrombus Region of Interest in data acquired with a "TI Scout" sequence. The potential loss of contrast between blood pool and thrombus is clearly seen.

\section{Figure 2}

Signal evolution with inversion time for blood pool and thrombus Region of Interest in data acquired with a "TI Scout" sequence. The potential loss of contrast between blood pool and thrombus is clearly seen. 


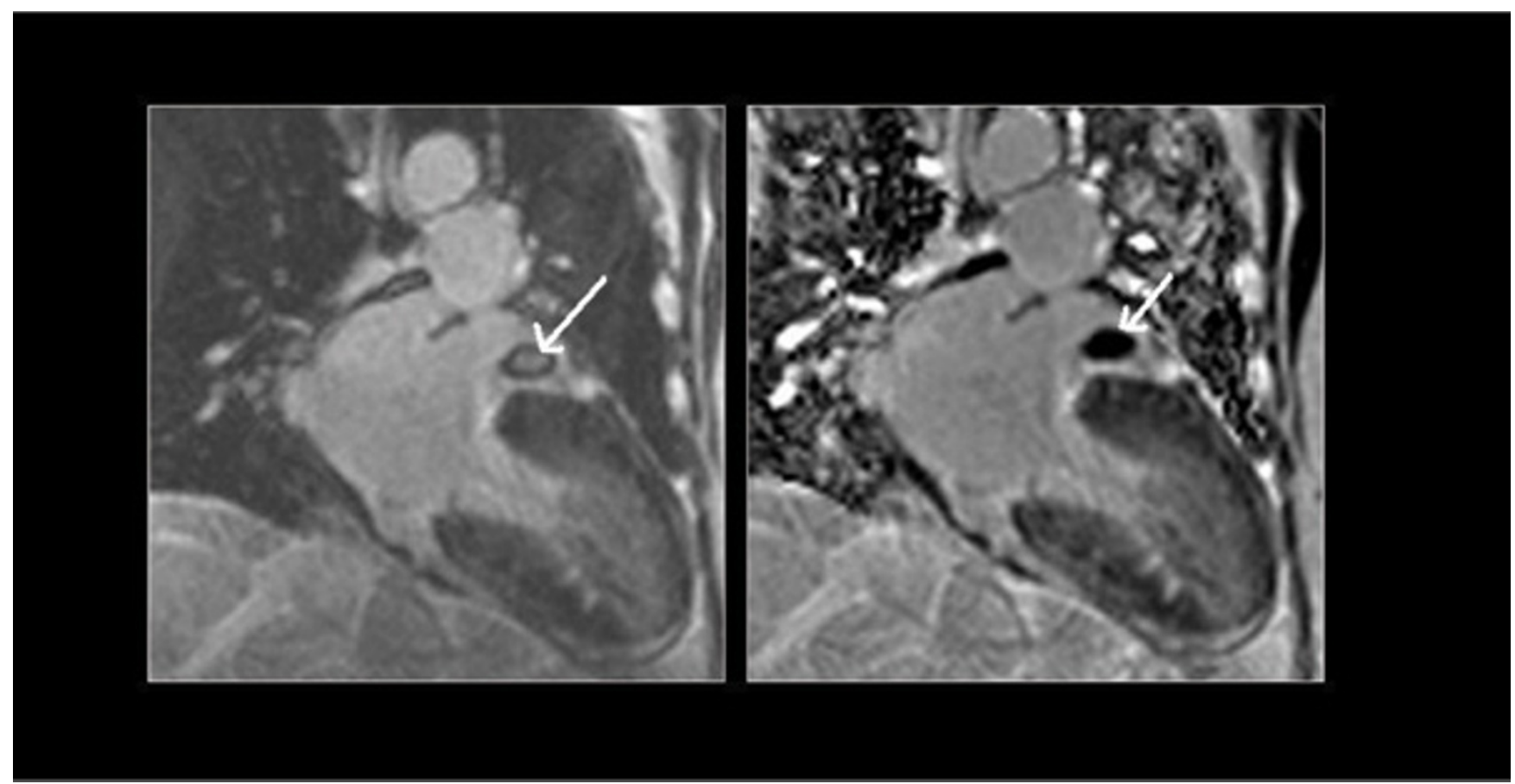

Figure 3

Magnitude (left) and PSIR (right) recontructed images of a long axis veiw of a patient with Left Atrial Appendage thrombus. The higher contrast in the PSIR is seen though the Magnitude reconstruction shows the typical "black rim" around the area in voxels on the boundary between tissues with positive and negative magnizaton (longer or shorter than the selected TI).

\section{Publish with Biomed Central and every} scientist can read your work free of charge

"BioMed Central will be the most significant development for disseminating the results of biomedical research in our lifetime. " Sir Paul Nurse, Cancer Research UK

Your research papers will be:

- available free of charge to the entire biomedical community

- peer reviewed and published immediately upon acceptance

- cited in PubMed and archived on PubMed Central

- yours - you keep the copyright 